\title{
Massive Dilation of the Ureter: An Endoscopic Management of Persistent Urinary Leak After Partial Nephrectomy
}

\author{
Panagiotis Pardalidis, MS, ${ }^{1}$ Nick Andriopoulos, MD, \\ Eleni Kosmaoglou, MD, PhD, and Nick Pardalidis, MD, PhD, FEBU ${ }^{2}$
}

\begin{abstract}
Introduction: The incidence of urinary leakage is increased as nephron sparing surgery becomes more used for the management of small renal masses. We present an endourologic approach in a patient with a persistent urinary leak after partial nephrectomy by placing two synchronous ureteral stents to improve drainage and avoid further potential surgery.

Patients and Method: A 39-year-old patient presented with a urine leak on the fifth postoperative day after an open partial nephrectomy. Initially, the leakage was managed with a placement of a 6F Double-J stent. However, due to persistence of leakage after a month, two ureteral stents (a $6 \mathrm{~F}$ Double-J stent and a $14 \mathrm{~F}$ endopyelotomy stent) were placed. With this maneuver, the ureter was dilated to $20 \mathrm{~F}$ and a bladder catheter was placed to complete the drainage.

Results: At 3 weeks, a CT urography was performed, which showed complete resolution of the urinary leakage compared with previous examination. The stents were removed the following day and the patient required no further intervention. The follow-up examination was continued for 24 months without any symptom of the patient and any sign of leak recurrence.
\end{abstract}

Conclusion: Endoscopic approach to urinary leak after partial nephrectomy obtaining high ureteral gauge can be a good alternative, avoiding more aggressive surgical approaches.

Keywords: partial nephrectomy, urinary leakage, endoscopic dilation of ureter

\section{Introduction and Background}

$\mathbf{P}$ ARTIAL NePHRECTOMY is the standard treatment for most T1a lesions.

Urinary fistula is a complication of partial nephrectomy, which ranges between $2 \%$ and $10 \%$ in most large series. ${ }^{1}$ The optimal management depends, mainly, on kind and amount of the leak, vascularization around the leak, and surgeon's preferences. Normally, ureteral stenting only can resolve the problem, but in $38 \%$ of the cases persistent urinary leakage can be a challenging complication. Prolonged ureteral stenting is the next step before proceeding to nephrostomy placement or surgical repair.

In our case, a persistent urine leak was effectively treated after placement of two different in diameter stents in the renal unit, in an effort to provide maximum drainage and therefore facilitate the closure of the fistula.

We describe this endoscopic technique of managing prolonged urinary leakage, before further intervention with more invasive procedures be considered.

\section{Case Presentation}

A 39-year-old patient with a history of Crohn's disease, presented with an asymptomatic $3 \mathrm{~cm}$ left renal tumor located at the lower pole, as a random finding in a CT scan examination. At the beginning of the procedure, the patient was placed in a lateral decubitus position and a $6 \mathrm{~F}$ open-end ureteral catheter was inserted in the renal unit, via flexible cystoscope, to check the water tightness of the collecting system

\footnotetext{
${ }^{1}$ Imperial School of Medicine, London, United Kingdom.

${ }^{2}$ Athens Medical Centre, Athens, Greece.
}

(C) Panagiotis Pardalidis et al. 2017; Published by Mary Ann Liebert, Inc. This is an Open Access article distributed under the terms of the Creative Commons Attribution License, which permits unrestricted use, distribution, and reproduction in any medium, provided the original work is properly cited. 


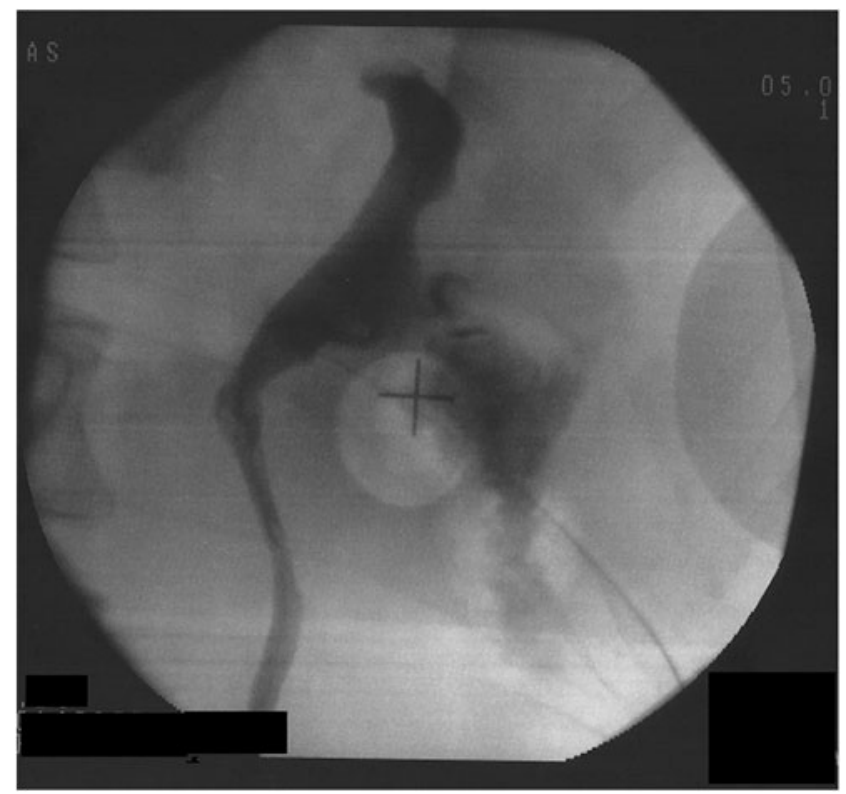

FIG. 1. Retrograde pyelography revealing the lower pole urinary leak.

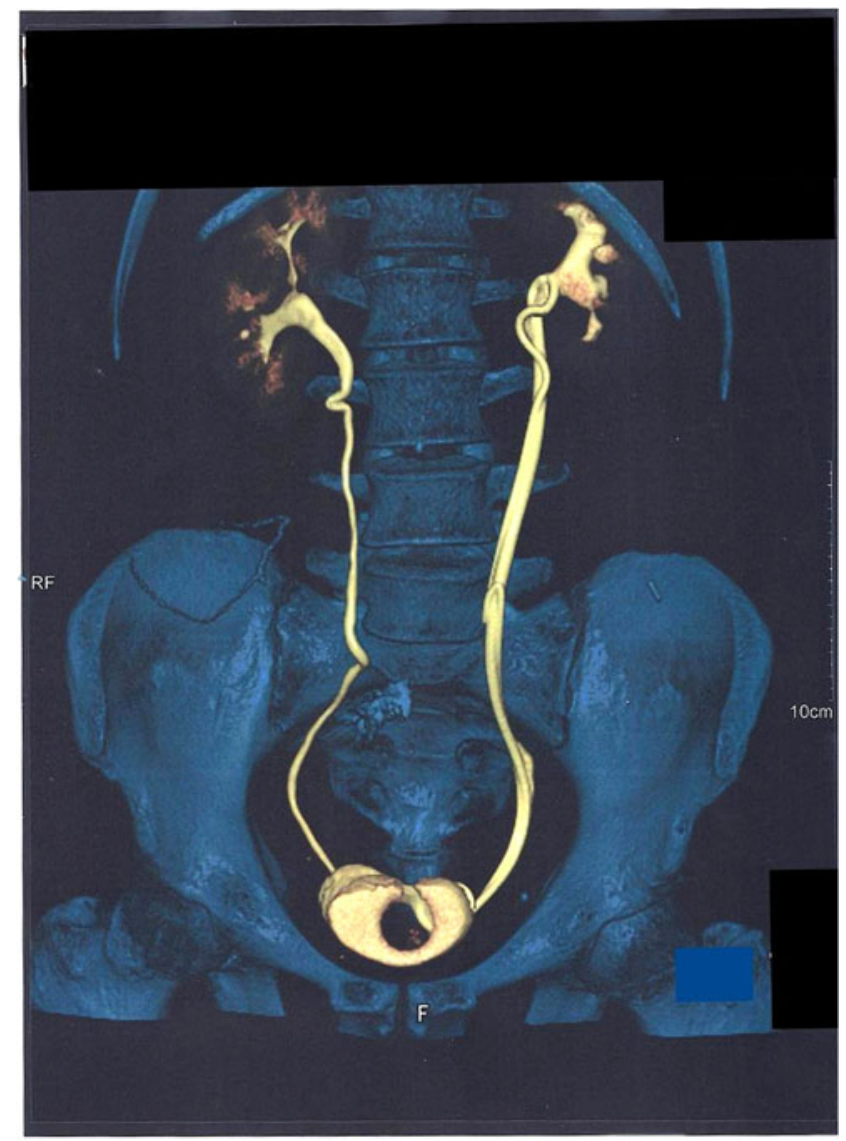

FIG. 2. CT urography after 3 weeks, before stent removal, showing no sign of urinary leak. after suturing. Then, the patient underwent partial nephrectomy, through extra peritoneal approach. The initial postoperative period was uneventful.

On the fifth postoperative day, the patient presented urine leak from the existing retroperitoneal suction drain.

Retrograde pyelography was performed revealing a lower pole leak and then a $6 \mathrm{~F}$ Double-J stent was placed in the renal unit along with continuous bladder drainage by a urethral catheter (Fig. 1).

Two weeks later the urine leak was still persistent, and we decided to perform double ureteral stenting using two different stents (a 6F Double-J stent and a 14/7F Retromax plus endopyelotomy stent) to achieve maximum dilation of the ureter up to $20 \mathrm{~F}$ in an effort to provide adequate drainage and therefore seal the leak. The two stents were directed in the kidney by gentle manipulation under fluoroscopic guidance, and finally, a urethral catheter was left in place. The patient was discharged from the hospital the next day.

Within 3 weeks after placement of the stents the leak from the drainage had ceased and a CT urography was performed, which showed complete healing and no sign of leak compared with the previous examination (Fig. 2).

A retrograde pyelography was performed, following the removal of the stents, which confirmed no sign of extravasation from the calix, on outpatient basis.

Follow-up examination with CT urography was performed at 12 and 24 months without any sign of leakage recurrence (Figs. 3 and 4).

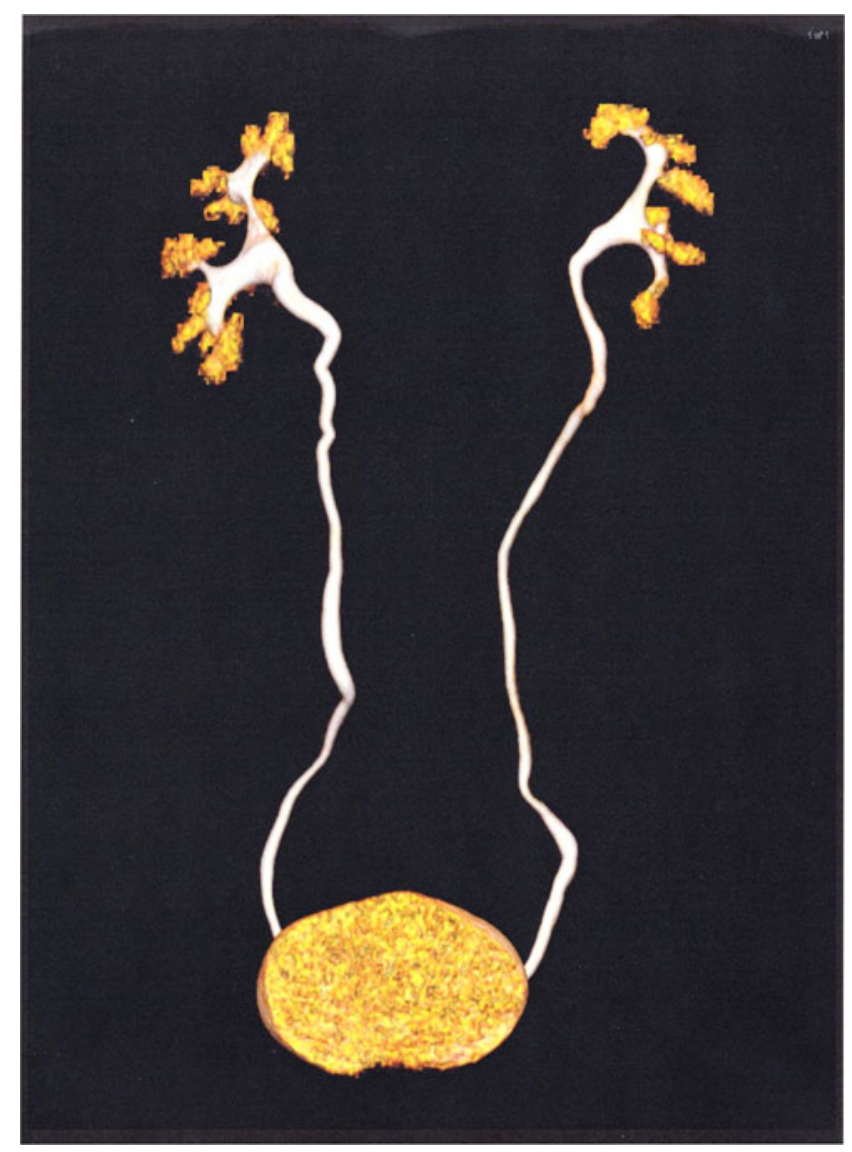

FIG. 3. CT urography at 12 months follow-up with no leak. 


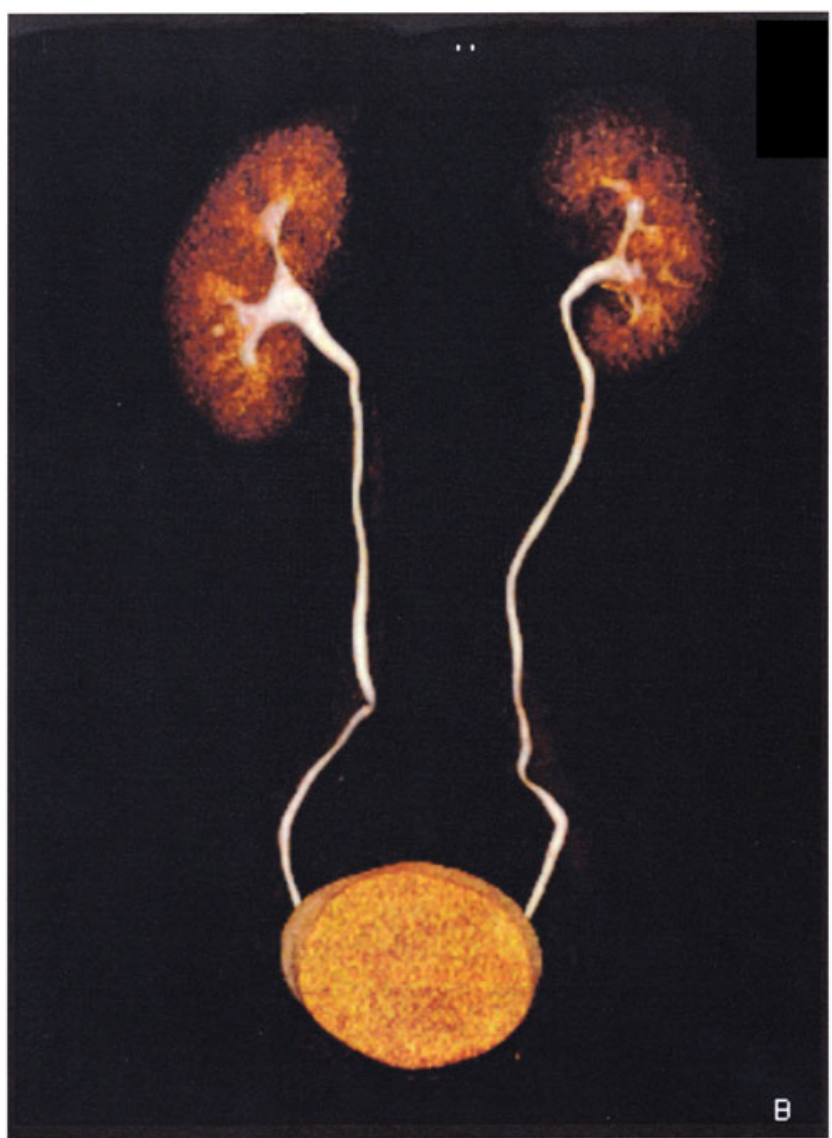

FIG. 4. CT urography at 24 months follow-up.

\section{Discussion and Literature Review}

Urine leakage is an undesirable complication after partial nephrectomy, which can usually resolve by drainage with a ureteral stent. However, in some cases, it is possible that a single stent may not be able to drain adequately. Most of the patients with urine leak will heal spontaneously with conservative treatment (drain, good nutrition, rest, and exercise to promote healing) in the absence of clinical symptoms. The decision to intervene is multifactorial and depends on patient decision, physician's preference, and the presence of clinical symptoms (flank pain, fever).

A number of different methods have been described for the management of persistent urine leak. Dual stents have been previously used as a method to prevent ureteral obstruction from extrinsic compression. Double drainage is a welldescribed alternative to manage prolonged urinary leakage.

Percutaneous drainage is usually used if endoscopic manipulations prove to be ineffective.

Girard et al. have proposed the use of a Malecot $16 \mathrm{~F}$ catheter to dilate the ureter by a retrograde approach, but this technique requires the ureter to be prepared using a Double-J stent. Another drawback is the length of the catheter, which can provoke occlusion. ${ }^{2}$

We strongly believe that dual stent placement offers wide dilation of the ureter and therefore can provide an optimal draining gauge, so it can be useful for cases of persistent urinary leak after nephron sparing surgery. This method provides adequate urinary drainage, facilitating rapid closure of the urine leak site and is well tolerated with only mild discomfort reported by the patient. ${ }^{3}$

Furthermore, it is possible that a single stent may be occluded by mucus and epithelial debris, therefore, it will not provide adequate extraluminar and intraluminar drainage to seal the urinary leak. ${ }^{4}$

The advantage of the technique we described using two stents with total diameter of $20 \mathrm{~F}$, is the maximum dilation of the ureter, to avoid percutaneous access or open surgical reintervention, with the drainage being exclusively performed endoscopically.

Conclusively, double-wide diameter ureteral dilation can be a good alternative in cases where single-stent drainage provides unsatisfactory results.

\section{Author Disclosure Statement}

No competing financial interests exist.

\section{References}

1. Simmons MN, Gill IS. Decreased complications of contemporary laparoscopic partial nephrectomy: Use of a standardized reporting system. J Urol 2007;177:2067-2073.

2. Girard F, Thanigasalam R, Theveniaud PE, et al. Prolonged urinary leakage after partial nephrectomy: A novel management pathway. Urology 2014;83:485-488.

3. Papatsoris A, Dellis A, Daglas G, et al. Management of JJ stent-related symptoms. Acta Chir Iugosl 2014;61:73-74.

4. Jarett TW, Lee CK, Pardalidis NP, Smith AD. Extensive dilation of distal ureter for endoscopic treatment of large volume ureteral disease. J Urol 1995;153:1214-1217.

Address correspondence to: Nick Pardalidis, MD, PhD, FEBU Athens Medical Center 6 Platonos Street Halandri Athens 15234

Greece

E-mail: npardali@otenet.gr

Cite this article as: Pardalidis $\mathrm{P}$, Andriopoulos N, Kosmaoglou E, Pardalidis N (2017) Massive dilation of the ureter: an endoscopic management of persistent urinary leak after partial nephrectomy, Journal of Endourology Case Reports 3:1, 186-188, DOI: 10.1089/ cren.2017.0102. 